\title{
"We're Actually More of a Likely Ally than an Unlikely Ally": Relationships Between Syringe Services Programs and Law Enforcement
}

\section{Carol Y. Franco ( $\nabla$ carol.franco@cuanschutz.edu )}

University of Colorado Anschutz Medical Campus https://orcid.org/0000-0003-4095-4696

\section{Angela Lee-Winn}

University of Colorado Anschutz Medical Campus: University of Colorado Denver - Anschutz Medical Campus

\section{Sara Brandspigel}

University of Colorado Anschutz Medical Campus: University of Colorado Denver - Anschutz Medical Campus

\section{Musheng Alishahi}

University of Colorado Anschutz Medical Campus: University of Colorado Denver - Anschutz Medical Campus

\section{Ashley Brooks-Russell}

University of Colorado Anschutz Medical Campus: University of Colorado Denver - Anschutz Medical Campus

\section{Research}

Keywords: syringe service programs, needle exchange, law enforcement, harm reduction, qualitative research

Posted Date: October 16th, 2020

DOl: https://doi.org/10.21203/rs.3.rs-91531/v1

License: (c) (1) This work is licensed under a Creative Commons Attribution 4.0 International License. Read Full License

Version of Record: A version of this preprint was published at Harm Reduction Journal on August 4th, 2021. See the published version at https://doi.org/10.1186/s12954-021-00515-2. 


\section{Abstract}

Syringe Services Programs (SSPs) provide sterile needles and a range of health services (e.g., HIV and HEP-C testing, overdose prevention education, provision of naloxone) to a hard-to-reach population, including people who inject drugs (PWID), aiming to prevent the transmission of infectious diseases. We performed a qualitative needs assessment of existing SSPs in the state of Colorado in 2018-2019 to describe the SSP activities, needs, and barriers. We performed semi-structured interviews with key program staff of SSPS $(n=11)$. All interviews were digitally recorded, transcribed, and validated. Qualitative researchers coded each transcript and maintained coding consistency through coding statistics (Kappa coefficient > 0.80) between coders. Memos were written to synthesize main themes. Nearly all the SSPs discussed their relationships with law enforcement at length. All SSPs viewed having a positive relationship with law enforcement as critical to the success of their program. Main factors that influence the quality of relationships between SSPs and law enforcement included: 1) alignment in agency culture, 2) support from law enforcement leadership, 3) police officers' participation and compliance with the Law Enforcement Assisted Diversion (LEAD) program, which provides intensive case management for low-level drug offenders, and 4) implementation of the "Needle-Stick Prevention Law" and Drug Paraphernalia Law Exemption. All SSPs expressed a strong desire to have positive relationships with law enforcement and described how a collaborative working relationship was critical to the success of their programs. Our findings suggest effective strategies to foster relationships between SSPs and law enforcement as well as key barriers to address.

\section{Introduction}

Syringe Service Programs (SSPs), also commonly referred to as syringe exchange, syringe access, or needle exchange programs, aim to prevent the transmission of infectious diseases by providing clean needles and a range of other services to people who inject drugs (PWID) and have shown to be effective in reducing the spread of viral hepatitis and HIV (Aspinall et al., 2014; Center for Disease Control \& Prevention, 2016; Fernandes et al., 2017; Martin, Hickman, Hutchinson, Goldberg, \& Vickerman, 2013; Platt et al., 2017).

In the United States, many SSPs were started in defiance of state laws banning the distribution of clean needles. For example, in Colorado, the setting of our study, the first syringe exchange program began in 1989, as a non-state sanctioned initiative to address the spread of HIV in one county (Asmar, 2009). Unlawful distribution of clean needles continued until 2010, when the Colorado Governor signed a law legalizing these programs statewide ( C.R. S. §25-1-520, Colorado.gov, 2018). Given these beginnings, it is not surprising to see discordance between SSP activities and law enforcement practices. Despite the growing prescence of SSPs, currently operating in 39 states and D.C. (Kaiser Family Foundation, 2018), there remains variation in state laws restricting the purchase and possession of sterile syringes (LawAtlas, 2019). For instance, some states have decriminalized syringe possession or purchase while others have allowed exemptions from drug paraphernalia charges (LawAtlas, 2017). 
Prior studies have described how law enforcement actions can have a negative influence on the ability of a SSP to provide services (Beletsky, Grau, White, Bowman, \& Heimer, 2011; Small, Kerr, Charette, Schechter, \& Spittal, 2006). This could be by direct interference with operations and access to SSPS or through other practices such as charging people who use drugs with paraphenalia in contradiction to the law (Bluthenthal, Kral, Erringer, \& Edlin, 1999; Cohen \& Csete, 2006; Lazzarini, Bray, \& Burns, 2002; Small et al., 2006). Negative interactions with law enforcement can deter PWID from using SSP services (Beletsky et al., 2015). One reason for law enforcement practices that undermine harm reduction programs could be lack of awareness on the part of law enforcement and perception that the harm reduction approaches are counterproductive (Beletsky, Macalino, \& Burris, 2005; Sightes et al., 2019; Wodak \& McLeod, 2008). There is also evidence that law enforcement, when supportive of public health efforts, can facilitate participation by referring PWID into the program (DeBeck et al., 2008). Identifying effective means of communication, including having tailored in-service training with police officers have proven effective in obtaining buy-in from law enforcement (Sightes et at., 2019; Strike \& Watson, 2017).

Given the importance of the role of law enforcement professionals in the implementation of successful SSPs, we sought to better understand the nature of current relationship and factors that facilitate and hinder a collaborative relationship and adoption of a harm reduction model. This study builds on the existing literature in the context of a U.S. state with exemptions for drug paraphernalia charges.

\section{Methods}

We conducted semi-structured interviews with key program staff at all legislated syringe service sites in the state of Colorado $(n=11)$. As of 2019, there were 11 programs operating in eight counties across the state. The interviews were part of a broader needs assessment of currently operating programs. We developed a semi-structured interview guide to understand how SSPs define, determine and/or measure their impact on the service population; SSPs' knowledge of community perceptions of their program and services they provide; SSPs' communication and/or collaboration with boards of health, county commissioners/city council, district attorneys, and law enforcement.

We received contact information for the primary contact for each SSP from the Colorado Department of Public Health and Environment (CDPHE). Primary contacts included program coordinators, directors, and health educators. Interviews were conducted either in-person $(n=9)$ or by videoconference $(n=2)$ depending on availability of the interviewee and their location. In-person interviews were recorded using a digital voice recorder (Olympus WS-852), phone interviews were recorded through a digital recorder or Talk Helper Call Recorder, and videoconference interviews were recorded using the Zoom application. Interviews lasted between 37 minutes to 1 hour and 54 minutes. The interviews were led by members of the research team with experience in collective qualitative data (CF, SB, MA). We conducted interviews using broad lines of questioning and introduced additional questions during the interview process as relevant information was revealed by the interviewee. Interview recordings were professionally transcribed via a third-party (TranscribeMe, San Francisco, CA), validated by members of the research team, and analyzed with qualitative analysis software NVivo 11 (QSR International Pty Ltd, Doncaster, Victoria). 
Two independent research team members (CF, AL) coded the transcripts individually and coding consistency checks were done between coders (Kappa coefficient $>0.80$, McHugh, 2012) and resolved discrepancies through discussion. We also wrote memos for each transcript summarizing major themes that addressed the goals of the interviews, in addition to other topics that emerged during the interviews. Data collection, analysis, and reporting followed guidelines established by Consolidated Criteria for Reporting Qualitative Research (COREQ; Tong, Sainsbury, \& Craig, 2007). We present supporting quotes from SSPs indented and in italics.

The data collection procedures were reviewed by the Colorado Multiple Institutional Review Board (COMIRB) and determined to not be human subjects research and reviewed by the Institutional Review Board at the CDPHE.

\section{Results}

\section{Overview of Variation in Relationships with Law Enforcement}

All SSP staff members discussed at length the relationship with law enforcement. All SSPs viewed having a positive relationship with law enforcement as important to the success of their program and having a negative or non-existent relationship with law enforcement as a barrier to the program and potentially having a negative impact on SSP clients. The degree of engagement and the type of collaboration with law enforcement varied by site. Relationships ranged from having a partnership with a high degree of interactions from both parties, having no relationship (evidenced by little coordination or communication), to having a hostile or confrontational relationship.

\section{Value of Relationships}

Programs that described having a positive relationship shared that the partnership had positive implications for SSPs and clients including facilitating referrals of clients into social services, working with SSPs to reduce syringe litter, and reducing re-offenses in the criminal justice system. For example, in one community the law enforcement referred PWID to social services and programs when they encounter them through their normal duties. In another community, law enforcement partnered with the SSP to hold a syringe litter pick-up day, whereas in other communities there is lack of clarity on who can legally provide pick-up services. SSPs that reported a lack of collaboration with law enforcement expressed a desire to do so and acknowledged the potential benefits of having a positive relationship including being able to learn from each other and working together to best serve substance users in their community.

"Yeah. So we have had very longstanding good relationships with the district attorney's office and with law-enforcement agencies and with other service-oriented providers in the community. We work very closely with them on trying to do a comprehensive approach to substance use. And so when we span the full spectrum, from prevention, early intervention, treatment, harm-reduction, we try to have a-- we're trying to have a collective impact approach where we're reducing the negative impact of substance use in 
general. And harm reduction is at that table along with law enforcement, treatment, prevention, healthcare-everyone at the table." - Site 1

"For me, ideally, I would like to see a relationship where we can do like reciprocal training. And so we can go in and talk to people about like, these are the ways that work effectively to communicate with people who are or like exhibiting a bad experience with drugs or things like that or these are the ways to get people on board with you. And in reciprocal training, I would love for law enforcement to come in and teach our program a little bit about-what are the things that we can teach our participants [SSP clients] to do to keep themselves a little less in the public eye? And I think police also have the opportunity to give like trauma-informed training and some of those things. So I would love to see some collaboration that way." - Site 2

\section{Factors that Influence Quality of Relationships}

The main factors that influenced the quality of relationships between SSPs and law enforcement included 1) alignment in agency cultures in adopting a harm reduction model, 2) support from law enforcement leadership, 3) police officers' participation and compliance with the Law Enforcement Assisted Diversion (LEAD) program, and 4) variation in the implementation of laws, including the "NeedleStick Prevention Law." Ongoing communication between SSPs and law enforcement was a critical factor in all aspects of maintaining a positive relationship with law enforcement agencies.

\section{Alignment in Agency Culture}

Almost all SSP site staff mentioned that law enforcement agency culture, beliefs, or attitudes towards harm reduction approaches influenced law enforcement perception of SSP services, the relationship between SSPs and law enforcement, and had implications for SSP clients. Some SSP staff expressed that law enforcement's positive endorsement of the harm reduction model and an agency culture marked by reduced biases towards people with substance use dependence supported a positive relationship between SSPs and law enforcement agencies. The partnership then led to law enforcement providing more support and resources for SSP clients, specifically PWID. Alignment in attitudes and beliefs consisted of law enforcement officers viewing SSPs as a resource for PWID to reduce the spread of disease, access treatment, and reduce the likelihood of needle-stick injuries for law enforcement. Those who did not share this belief viewed SSPs as a means to enable substance use and accelerate syringe litter in their community.

Through a shared philosophy of harm reduction, either through a collective agency culture or by individual police officers, SSPs and law enforcement were able to have a positive working relationship. There were several examples of how SSP and law enforcement could work together. For example, some SSPs conducted trainings for law enforcement and educated them on the benefits of using a harm reduction model to address substance use and the spread of disease. These trainings led to a change in perspective among law enforcement officers and assisted in positive collaboration and/or engagement. One SSP site has representation of law enforcement on their board of directors and as a result, has police 
officers regularly visit the site for tours and law enforcement refer potential PWID clients to the SSP when on duty. Another SSP observed that law enforcement representatives attend opioid prevention coalition meetings. Some sites noted that having an established positive relationship with law enforcement leads to better communication. For example, one site described that they shared regular program reports with law enforcement leadership to keep them informed about the program.

"They [law enforcement] worked really closely with us in learning about the Law Enforcement-Assisted Diversion program, and they are now harm-reduction advocates themselves. And I am sometimes am in a meeting and it's the [law enforcement] staff who are correcting people about harm reduction [laughter]. Or not correcting people, but bringing to people's attention like, "Oh, the only end goal is not treatment. We also need to think about harm reduction and keeping people safe when they're not actively using... And it's coming from law enforcement, not from me. So I think that we not only have good relationships, but we've also infiltrated [laughter]. I don't know if infiltrated is the right term, but you know what I mean. They've also adopted a harm-reduction philosophy." - Site 1

"But then also when we had the community meetings, we had some law enforcement officers at those meetings, and they were concerned about getting more needles out into the community and the parks and whatever. And we had some staff from CDPHE [Colorado Department of Public Health and Environment] on the phone addressing those concerns. And it seems like after that, they were more supportive of the program, at least the Chief of Police and the Sheriff. I'm sure there's some law enforcement that aren't sold on the program and that have some concerns or reservations. But I think for the most part, once we got the Chief of Police onboard and the Sheriff, then it was like, "Well, this is how it's going to go." And so we did get that buy-in. And initially, as we were doing the community meetings and educating the Board of Health and all of that, it was like, "Oh, no, we don't need more syringes in our parks." But then, when they heard from other sites around the state and from the health department, then it was like, "Oh, yeah. Okay. Well, I see the point. The point is to get them off the street and for people to stay healthier." - Site 3

A few SSP sites mentioned law enforcement's labeling of SSPs as "enablers" and that this was a common and problematic perception. Others mentioned that law enforcement's preexisting negative perceptions towards PWID and SSPs often lead law enforcement to ignore the state-wide law to protect SSP clients. A few SSPs attributed such negative law enforcement culture to misunderstanding of SSP' role in the community. The belief that SSPs are "enablers" of continued drug use or directly providing illegal substances, such as fentanyl, were common misperceptions. Interviewees suggested this is more common in law enforcement agencies having a "conservative" agency culture. One SSP participant, for example, mentioned how one district attorney brought false and misleading evidence against the SSP to highlight how SAPs "enable" more drug use among SAP clients. These shared beliefs among law enforcement agencies have negative implications for SSP clients, as described by a few SSP sites, whereby having a conservative agency culture was believed to have contributed to the charges placed on SSP clients having syringes on their person, reluctance of clients to seek help from police or other emergency personnel when their or someone else's safety is endangered. 
"I think that the police departments don't always like syringe access programs... I think they views what we do is enabling and giving people the tools to do things illegally." - Site 2

"I think that there are quite a few officers who view us as enabling folks. We had one particular officer show up to our board of health meeting... And this officer just thinks that we're not limiting how many syringes that we give out, that we need to start marking our syringes. So we can be held accountable for the syringes in public... would say, on the whole, they think that we're a bad service... I don't think that they view at us as a spectrum of treatment or a spectrum of care. I think that a lot of it really boils down to the perception of the people who use drugs and that anybody who tries to help them is also bad." - Site 4

"All of our people feel terrified... They don't feel comfortable to call emergency services because they know that the cops are going to come. So they're taking on this person's life without any other assistance because they don't want to be punished. Saving a life shouldn't be criminalized, regardless of what you're doing." - Site 4

\section{Support from Law Enforcement Leadership}

Most sites discussing the role of law enforcement leadership discussed having positive relationships and that these relationships had implications for their program and the clients they are serving. Positive endorsement and/or a positive relationship with law enforcement leadership was often a result of ongoing communications between SSP sites and law enforcement leaders, such as the Chief of Police, who would serve as a champion for SSP sites and their mission in reducing the spread of disease. SSPS were able to receive support from leadership by communicating the goals of SSPs with leadership, educating leaders of the role of SSPs on a spectrum of care, and their efforts to reduce syringe litter. Law enforcement leaders who advocated for SSPs were perceived as influencing the overall law enforcement agency culture and influencing the attitudes, beliefs, and behaviors of individual officers. Partnerships between law enforcement leadership and SSP staff also led to additional training for officers, including laws surrounding harm reduction and paraphernalia charges to SSP clients.

"I have a Lieutenant with the [name redacted] Police Department on my board of directors. I have a good relationship with the Commander in this district and have a great relationship with the Chief of police. The Chief of Police used to be our commander over in District One and knows that we are pro-public safety. So that's been very helpful for us too, because some exchange programs don't work with law enforcement or law enforcement won't work with them. The Police Department has been very open to that and I think it's been beneficial for both of us. They don't sit out front [of our building]. We bring them in for tours in the afternoon. They refer more to my program than healthcare providers." - Site 5

"When we mentioned to them that we're seeing an uptick in people getting paraphernalia charges. They actually had in one of their chiefs meeting where all the chiefs of police come, they went and did a review of all the harm reduction laws that the officers need to be aware of and how they need to not be filing paraphernalia charges for participants[SSP client] of our program, and they are making sure that our local law enforcement are abiding to harm reduction laws." - Site 6 
"Our previous director had a really great relationship with the new chief, Chief [Last Name], which is fantastic. Chief [Last Name] seems to be mostly in support of what we're doing as far as being on the spectrum of treatment because you can't get somebody into treatment if they're dead. Right? You can't get someone into treatment if no one's engaging with them. So I think that's fantastic.... Get us in there so we can do some education with the new police officers. I think that it could be a really great partnership and we could change the narrative of how folks are treated because they're even taking syringes for naloxone because we provide injectable naloxone. They are taking those syringes. So it's how are they going to administer this medication and save someone because you are taking that syringe?" - Site 4

\section{LEAD Program Participation}

The Law Enforcement Assisted Diversion (LEAD) is a program that provides intensive case management for low-level drug offenders. Established in Seattle, WA, previous studies have shown how the LEAD results in a reduction in recidivism by diverting people to social services instead of incarceration (Clifasefi, Lonczak, \& Collins, 2017). The LEAD program offers an alternative route for low-level drug offenses that provides case management and connection with resources rather than jail and prosecution. The LEAD programs are operating in four counties in Colorado that also have SSPs (Colorado Department of Human Services, 2019), and nearly all these SSPs noted this in their interviews that LEAD is a positive program in their community. Some SSP staff noted that LEAD is part of a changing culture in law enforcement around responding to drug use. One SSP staff member expressed the helpfulness of the program in giving law enforcement more options than arresting and putting individuals in jail when what they often need is services and treatment. Another participant mentioned that the LEAD program is an opportunity to work in partnership with law enforcement, and that LEAD officers' presence in the community provides a feeling of safety among PWID.

"... But I think the officers here are more feeling like, "Oh, there's options we have besides just throwing someone in jail, they get back out, throwing them back in jail, they get back out." And so I think that has helped giving law enforcement options. I mean it's still their decision whether they take someone to jail or not. But just to know that there are some options and support that they have. That has been really helpful." - Site 3

"The interactions I've had with them have been primarily through the LEAD Program, the Law Enforcement Assisted Diversion. We've got an excellent working relationship with them. As a matter of fact, they've got folks that will come in here and volunteer fairly regularly. Again, that's an opportunity for them to make that face-to-face contact with the participants [SSP clients] within the program. They will wear their LEAD shirts and stuff in here. So that way, participants know that, "Hey, these guys are our reductionists. These guys aren't out to get you. These guys want to help you." - Site 7

Not all SSP participants however were successful at having buy-ins from law enforcement to implement the LEAD program in their communities. A few SSP participants acknowledged challenges of successfully implementing and disseminating the LEAD program in their communities, including less 
systemic adoption of the LEAD program and unknown reasons for lack of buy-in from law enforcement leadership.

"I think the officers here are more feeling like, "Oh, there's options we have besides just throwing someone in jail, they get back out, throwing them back in jail, they get back out." And so I think that has helped giving law enforcement options." - Site 3

"So there is that kind of negativity, but on the whole, the street cops get it. They'd rather not be messing with this either. They wanted to do LEAD, but the tops of law enforcement have set the exclusion criteria so strict that no one can really get into the program through them at that point, so. But that's that. That's the upper levels." - Site 8

\section{Varying Implementation of Laws}

Many sites shared that officers do not adhere to laws that relate to ticketing of people for drug paraphernalia. According to state statute titled the Drug Paraphernalia Law Exemption (C.R.S. §18-18-425 through 18-18-430, Colorado.gov, 2019) syringe exchange program clients are exempt from drug paraphernalia charges. A state Needle-Stick Prevention Law (C.R.S., §18-18-428, Colorado.gov, 2019) also allows for an exception to drug paraphernalia charges if someone informs a law enforcement officer, prior to search, that they have a sharp object.

SSPs provide cards to clients to show they are exempt from drug paraphernalia charges. However, SSP participants are still sometimes ticketed, either due to an apparent lack of familiarity of the law, or in some cases, in apparent defiance of the law. Several SSP staff shared that law enforcement do not adhere to the needle stick law due to their personal beliefs towards PWID. A couple sites noted the importance of framing the state statutes to protect law enforcement from being pricked by a used syringe while patting someone down. These sites shared that by presenting the law in a way that demonstrates its benefits to law enforcement officers, they would have more buy-in and adherence to the law by officers who have direct contact with SSP participants.

"So we've gotten really good buy in from them because we know that one-third of law enforcement officers nationally will be pricked by a syringe at some point in their career. Two-thirds of that one-third will be pricked multiple times. If we passed two pieces of statewide legislation that reduce needle stick injuries in law enforcement and promote proper disposal, they love that." - Site 5

"So that law is meant to- it's meant to protect them. And I think we'd best help if we had a symbiotic relationship with law enforcement." - Site 9

Among those sites who characterized their relationship with law enforcement as problematic, the key concern was around police officers ticketing their clients with paraphernalia charges. Despite having these regulations in place to protect SSP participants from being charged, program staff from several sites reported that clients had shown law enforcement their SSP Identification card (ID) and were still ticketed or charged for having paraphernalia. SSP staff talked about these incidents and how they 
supported clients in disputing these charges by contacting the district attorney's office on behalf of the clients.

Law enforcement's observance of the SSP ID cards varied by site, though their resistance to abiding by the Colorado statute were influenced by officers' personal views surrounding the statute. SSP staff from one site detailed instances where law enforcement did not adhere to the laws on SSP ID cards, and instead tore up the ID card during an encounter and continued to search and/or charge the client. Similarly, a different site reported that their client also experienced resistance from law enforcement, and despite efforts to engage with law enforcement, they continue to give out tickets because of their negative views toward substance use and PWID. Staff recounted an instance when officers were reported to dump out sharps containers to find syringes with residue in them, despite a SSP client having a SSP ID Card on them. Another site discussed how law enforcement has become more accepting of the law and while many officers still ticket clients, it has become less frequent than when their site first opened.

"We've had officers tear up our participants' [SSP client] syringe exchange cards, saying that harm reduction is not a real thing. This isn't legal. We knew what we were doing was wrong. There's just not a lot of positive encounters. It's like once they see a syringe card, then they're like, 'Okay, well, we want to search your car.' It's like, all right, well, I mean, our folks put everything in their sharps containers most of the time. They disclose where they have everything so that you're not at danger by sticking yourself. I feel like for the majority, our folks are trying to be flexible and trying to be understanding." - Site 10

"And I have been wracking my brain for the last-- I guess five months now, trying to figure out how to start fostering a better relationship with law enforcement. And I would say it's very intimidating. And I don't know the best way to go about it because, in some ways, they're experts in their field. And in a lot of ways, we're experts in our field. But why can't we work together? And I think that that's really tough. And we've gone on several cop dates before, where we're just going out for coffee being like, 'Hey. Can you help us understand? We're getting reports.' We'll take officer's names down and say, 'We're getting a lot of reports that you're writing paraphernalia tickets. Help us understand because you know that the laws are in place to protect these people.' And they don't agree with it. So they write the tickets." - Site 4

"When we first started it was, 'Yeah, the cops stopped me. They took this. They confiscated my card and all my gear and told me that doesn't mean anything." We still hear that, but rarely anymore. We actually have heard a few people tell us, "Oh yeah, I showed the cop my card and he said, 'Okay, fine, have a nice day, and left." So that we didn't hear for- it took a while, but we have heard a few of those. Mostly we don't hear that much right now, so we think most of the cops are saying, "Oh, okay," and understand that it is the law, that they can't confiscate those people's personal property just because they don't like what they're doing because there's a law that says, 'Yes, you can have this."' - Site 8

A drug paraphernalia charge creates a cascade of events for SSP clients that can lead to negative outcomes, such as potential jail time. SSP staff often conduct outreach to law enforcement and district attorneys regarding inappropriate ticketing.

Page 10/15 
"The frustrating piece about law enforcement is that sometimes we are trying to just do our jobs, and they try to get tangled in our crosshairs. And so they'll sit in their cars outside of our building, which means that our folks won't come in." - Site 2

"And it's like, 'Okay.' So we're educating our participants saying like, 'Notify the officers that you have sharps on your person and you won't get a residue charge.' And they're still doing it. So I would say that's really tricky." - Site 4

"We have people that get ticketed for paraphernalia, oh, [redacted], three to five times a week. And the DA [district attorney] is so over it, it's not even funny. It's gotten to the point where somebody shows them their card or something that they got from us, that's easily recognizable from us, like that has our logo on it and shows them the ticket, he's like, "Go down, talk to the girls, get a letter. I'll see you later." We're like, "Okay, come in, we'll write up the whole letter," and charges get dropped. They're just doing it, anyway. The DA knows the rules, we're all tired of it." - Site 10

\section{Discussion}

All study participants (SSP staff members) discussed their relationships with law enforcement, which ranged from having a positive collaborative relationship to adversarial. Participants described the implications of the relationship for SSP clients, including unnecessary harassment and arrests by law enforcement. Participants describing a positive relationship with law enforcement discussed how programs and law enforcement shared similar views towards harm reduction initiatives, the need to reduce the spread of disease, and importance of connecting SSP clients to resources. Law enforcement training or education in harm reduction, communication between SSP and law enforcement agencies, and having a champion within law enforcement leadership were all factors contributing to a positive relationship. Lack of a relationships was attributed to a lack of buy-in from law enforcement leadership and officers including a conservative political perspective and a lack of interest in establishing a relationship from leadership.

\section{Implications for policy and practice}

Consistent with studies done in other communities in the U.S., our findings suggest several activities or strategies to foster relationships between police and SSPs, including SSPs to provide trainings, or encourage training, to law enforcement personnel on harm reduction models. Findings of prior research that trainings on occupational safety information (e.g. needle-stick prevention) is acceptable to police and is associated with a reduction in needle-stick injuries among law enforcement (Beletsky, Agrawal, et al., 2011; Groseclose et al., 1995). Future research could examine the trainings and training approaches that are most successful at supporting working relationships between SSP and with law enforcement. SSP can further demonstrate their commitment to supporting law enforcement by advocating for socalled needle stick laws. This is a discrete opportunity for SSPs to demonstrate they have the best interest of law enforcement officers in mind and can be advocates for law enforcement interests. A 
continuing focus on reducing syringe-related risks to law enforcement may be a pathway to demonstrate shared values.

Our findings suggest law enforcement professionals' existing attitudes towards PWID may supersede their adherence to the laws on SSP ID cards and result in inappropriate ticketing and charges. This calls for additional research on law enforcement professionals' attitudes and beliefs towards SSPs and PWID as well as their current training and understanding of the laws on SSP ID cards to expand our knowledge on building a successful working relationship between SSPs and law enforcement.

Finally, we found the adoption of the LEAD program is associated with a successful and supportive relationship between SSP and law enforcement. The causal direction of this is unclear; it seems likely that it is bidirectional in that more supportive police districts choose to adopt the LEAD program which then reinforces a harm reduction approach.

\section{LIMITATIONS}

Our findings are limited to existing SSPs in the State of Colorado; having data from one state with its unique community and legislative context may limit generalizability to states with differing policies. Detailed and nuanced results of our study however can inform practices and policies in other states with existing SSPs or are in the process of establishing a SSP in their community. Further research from the perspectives of law enforcement could also beneficial as it may vary from our findings from the perspective of SSPS.

\section{Conclusion}

All SSPs expressed a strong desire to have positive relationships with law enforcement and described how a collaborative working relationship was critical to the success of their programs. Not all SSPS however were fortunate in having a positive working relationship with law enforcement. Our findings suggest effective strategies to foster relationships between SSPs and law enforcement as well as key barriers to address. Our findings support the need for both SSPs and law enforcement to devote time and resources to build a strong, positive partnership. Having such positive relationships with law enforcement has implications for SSP clients, in that law enforcement will be less likely to ticket persons who get their syringes from SSPs, encourage PWID to seek services from SSPs, which can then lead them to other resources, such as housing, wound care, and substance use treatment programs.

\section{Declarations}

\section{Funding:}

This study was supported by a contract (PI: Brooks-Russell) from the Colorado Department of Public Health and Environment. The funding sources had no role in the interpretation of the data, preparation and of the manuscript or decision to submit the manuscript for publication. 


\section{Disclosure of potential conflicts of interest:}

The authors declare no conflicts of interest.

\section{Ethics Approval:}

This study was declared non-human subjects research and all procedures were in accordance with Colorado Multiple Institutional Review Board ethical standards. The authors certify that the study was performed in accordance with the ethical standards as laid down in the 1964 Declaration of Helsinki and its later amendments or comparable ethical standards. All participant and site identifying information was removed for anonymity.

\section{Informed Consent:}

Consent was not obtained by participants as this study was deemed non-human subjects research and was therefore not a requirement by Colorado Multiple Institutional Review Board.

\section{Declaration of Interest: None.}

Funding/Support: This study was supported by a contract (PI: Brooks-Russell) from the Colorado Department of Public Health and Environment.

Role of the Sponsor: The funding sources had no role in the interpretation of the data, preparation and of the manuscript or decision to submit the manuscript for publication.

Acknowledgements: We would like to acknowledge the colleagues at CDPHE and the Syringe Services Programs who contributed to study design or data collection.

\section{References}

Asmar, M. (2009). Why doesn't Colorado get the point of needle exchange programs? Westword. Retrieved from https://www.westword.com/news/why-doesnt-colorado-get-the-point-of-needle-exchange-programs5103306

Aspinall, E. J., Nambiar, D., Goldberg, D. J., Hickman, M., Weir, A., Van Velzen, E., . . Hutchinson, S. J. (2014). Are needle and syringe programmes associated with a reduction in HIV transmission among people who inject drugs: a systematic review and meta-analysis. International Journal of Epidemiology, 43(1), 235-248.

Beletsky, L., Agrawal, A., Moreau, B., Kumar, P., Weiss-Laxer, N., \& Heimer, R. (2011). Police training to align law enforcement and HIV prevention: preliminary evidence from the field. American Journal of Public Health, 101(11), 2012-2015. 
Beletsky, L., Cochrane, J., Sawyer, A. L., Serio-Chapman, C., Smelyanskaya, M., Han, J., ... \& Sherman, S. G. (2015). Police encounters among needle exchange clients in Baltimore: drug law enforcement as a structural determinant of health. American Journal of Public Health, 105(9), 1872-1879.

Beletsky, L., Grau, L. E., White, E., Bowman, S., \& Heimer, R. (2011). The roles of law, client race and program visibility in shaping police interference with the operation of US syringe exchange programs. Addiction, 106(2), 357-365.

Beletsky, L., Macalino, G. E., \& Burris, S. (2005). Attitudes of police officers towards syringe access, occupational needle-sticks, and drug use: a qualitative study of one city police department in the United States. International Journal of Drug Policy, 164(4), 267-274.

Bluthenthal, R. N., Kral, A. H., Erringer, E. A., \& Edlin, B. R. (1999). Drug paraphernalia laws and injectionrelated infectious disease risk among drug injectors. Journal of Drug Issues, 29(1), 1-16.

Centers for Disease Control \& Prevention. (2016). HIV and injection drug use: Syringe services programs for HIV prevention. Vital Signs.

Clifasefi, S. L., Lonczak, H. S., \& Collins, S. E. (2017). Seattle's Law Enforcement Assisted Diversion (LEAD) program: Within-subjects changes on housing, employment, and income/benefits outcomes and associations with recidivism. Crime \& Dlinquency, 63(4), 429-445.

Cohen, J., \& Csete, J. (2006). As strong as the weakest pillar: Harm reduction, law enforcement and human rights. International Journal of Drug Policy, 17(2), 101-103.

Colorado Department of Human Services. (2019, August, 2019). Law Enforcement Assisted Diversion (LEAD) Program. Retrieved from https://www.colorado.gov/pacific/cdhs/law-enforcement-assisteddiversion-lead-program

Colorado.gov. (2018). Colorado Revised Statues 2018. Retrieved from http://leg.colorado.gov/sites/default/files/images/olls/crs2018-title-25.pdf.

Colorado.gov. (2019). Colorado public health harm reduction legislation. Retrieved from https://www.colorado.gov/pacific/cdphe/colorado-public-health-harm-reduction-legislation.

DeBeck, K., Wood, E., Zhang, R., Tyndall, M., Montaner, J., \& Kerr, T. (2008). Police and public health partnerships: Evidence from the evaluation of Vancouver's supervised injection facility. Substance Abuse Treatment, Prevention, and Policy, 3(1), 11.

Fernandes, R. M., Cary, M., Duarte, G., Jesus, G., Alarcão, J., Torre, C., . . Carneiro, A. V. (2017). Effectiveness of needle and syringe Programmes in people who inject drugs-An overview of systematic reviews. BMC Public Health, 17(1), 309. 
Groseclose, S. L., Weinstein, B., Jones, T. S., Valleroy, L. A., Fehrs, L. J., \& Kassler, W. J. (1995). Impact of increased legal access to needles and syringes on practices of injecting-drug users and police officers-Connecticut, 1992-1993. Journal of Acquired Immune Deficiency Syndromes and Human Retrovirology: Official Publication of the International Retrovirology Association, 10(1), 82-89.

Kaiser Family Foundation. (2018). State Health Fscts: Sterile Syringe Exchange Programs.

LawAtlas. (2017, July 1, 2017). Syringe Distribution Laws. Retrieved from http://lawatlas.org/datasets/syringe-policies-laws-regulating-non-retail-distribution-of-drug-parapherna LawAtlas. (2019, August 1, 2019). Syringe Service Program Laws. Retrieved from http://lawatlas.org/datasets/syringe-services-programs-laws

Lazzarini, Z., Bray, S., \& Burns, S. (2002). Evaluating the impact of criminal laws on HIV risk behavior. The Journal of Law, Medicine \& Ethics, 30(2), 239-253.

Martin, N. K., Hickman, M., Hutchinson, S. J., Goldberg, D. J., \& Vickerman, P. (2013). Combination interventions to prevent HCV transmission among people who inject drugs: modeling the impact of antiviral treatment, needle and syringe programs, and opiate substitution therapy. Clinical Infectious Diseases, 57(suppl_2), S39-S45.

McHugh, M. L. (2012). Interrater reliability: the kappa statistic. Biochemia Medica: Biochemia Medica, 22(3), 276-282.

Platt, L., Minozzi, S., Reed, J., Vickerman, P., Hagan, H., French, C., . . Maher, L. (2017). Needle syringe programmes and opioid substitution therapy for preventing hepatitis $\mathrm{C}$ transmission in people who inject drugs. Cochrane Database of Systematic Reviews, 9.

Sightes, E., Ray, B., Paquet, S. R., Bailey, K., \& Huynh, P. (2019). Police officer attitudes towards syringe service programming. Drug and Alcohol Dependence, 205, 107617.

Small, W., Kerr, T., Charette, J., Schechter, M. T., \& Spittal, P. M. (2006). Impacts of intensified police activity on injection drug users: Evidence from an ethnographic investigation. International Journal of Drug Policy, 17(2), 85-95.

Strike, C., \& Watson, T. M. (2017). Relationships between needle and syringe programs and police: an exploratory analysis of the potential role of in-service training. Drug and Alcohol Dependence, 175, 51-54.

Tong, A., Sainsbury, P., \& Craig, J. (2007). Consolidated criteria for reporting qualitative research (COREQ): a 32-item checklist for interviews and focus groups. International Journal for Quality in Health Care, 19(6), 349-357.

Wodak, A., \& McLeod, L. (2008). The role of harm reduction in controlling HIV among injecting drug users. AIDS (London, England), 22 Suppl 2(Suppl 2), S81-S92. doi:10.1097/01.aids.0000327439.20914.33 